\title{
Near Infra-Red Phosphorescent Dinuclear Ir(III) Complex Exhibiting Unusually Slow Intersystem Crossing and Dual Emissive Behavior
}

Marsel Z. Shafikova,b,*, Andrey V. Zaytsevc, Alfiya F. Suleymanovad ${ }^{\mathrm{d}}$, Fabian Brandla ${ }^{\mathrm{a}}$, Aleksandra Kowalczyk $^{\mathrm{e}}$, Magdalena Gapińska ${ }^{\mathrm{f}}$, Konrad Kowalski ${ }^{\mathrm{g}}$, Valery N. Kozhevnikov ${ }^{\mathrm{c} *}$ and Rafał Czerwieniec ${ }^{\mathrm{a} *}$

a Institut für Physikalische und Theoretische Chemie, Universität Regensburg, Universitätsstrasse 31, Regensburg, D-93053, Germany.

${ }^{b}$ Department for Technology of Organic Synthesis, Institute of Chemical Technology, Ural Federal University, Mira 19, Ekaterinburg, 620002, Russia

c Department of Applied Sciences, Northumbria University, Newcastle upon Tyne, NE1 8ST, U.K.

${ }^{d}$ Department of Chemistry, University of York, Heslington, York YO10 5DD, UK.

${ }^{e}$ Department of Microbial Genetics, Faculty of Biology and Environmental Protection, University of Łódź, Banacha 12/16, 90-237, Łódź, Poland.

${ }^{f}$ Laboratory of Microscopic Imaging and Specialized Biological Techniques, Faculty of Biology and Environmental Protection, University of Łódź, Banacha 12/16, 90-237, Łódź, Poland.

${ }^{g}$ Faculty of Chemistry, Department of Organic Chemistry, University of Łódź, Tamka 12, 91-403 Łódź, Poland. 
DFT optimized ground state $\left(\mathrm{S}_{0}\right)$ and $\mathrm{T}_{1}$ state geometry of IrIr' in cartesian (XYZ) coordinates

\begin{tabular}{|c|c|c|c|c|c|c|c|}
\hline \multicolumn{4}{|c|}{ State $S_{0}$} & \multicolumn{4}{|c|}{ State $T_{1}$} \\
\hline $\mathrm{C}$ & -3.320607000 & 0000 & -3.714828000 & C & 3.279638000 & 65000 & -3.714838000 \\
\hline$c$ & -3.396805000 & 2.003855000 & -2.372920000 & $C$ & 3.358519000 & -2.027945000 & 9000 \\
\hline $\mathrm{N}$ & -3.643823000 & 0.713654000 & -2.025374000 & 14 & 3.628170000 & -0.742327000 & -2.025423000 \\
\hline$C$ & -3.795997000 & -0.194231000 & -2.979972000 & C & 3.803140000 & 0.163176000 & -2.978065000 \\
\hline$C$ & -3.728910000 & 0.105435000 & -4.322541000 & C & 3.734526000 & -0.135034000 & -4.320644000 \\
\hline$C$ & -3.487413000 & 1.420564000 & -4.720066000 & C & 3.467745000 & -1.445318000 & -4.718867000 \\
\hline C & -3.231257000 & 2.900115000 & -1.237843000 & $C$ & 3.176774000 & -2.923122000 & -1.238148000 \\
\hline C & -2.940339000 & 4.259266000 & -1.204290000 & $C$ & 2.863210000 & -4.277621000 & -1.205983000 \\
\hline C & -2.785150000 & 4.939774000 & 0.001781000 & $C$ & 2.695832000 & -4.955667000 & 0.000010000 \\
\hline C & -2.943266000 & & & C & & & \\
\hline C & -3.234280000 & 2.898885000 & 1.2 & C & 3.17 & -2.5 & 000 \\
\hline C & -3.358885000 & 000 & -0.0 & $c$ & 3.31 & -2.2 & \\
\hline C & -3.402910000 & 1000 & 2.3 & C & 00 & -2.027935000 & 00 \\
\hline C & -3.330243000 & 2.360173000 & & C & 00 & -2.38 & \\
\hline C & -3.499892000 & 1.415946000 & 4.7 & C & 000 & -1.445299000 & 00 \\
\hline C & -3.740584000 & 0.101246000 & 4.3 & C & 000 & -0.135017000 & 4.32 \\
\hline C & -3.804122000 & -0.19 & & . & 0000 & 88000 & 000 \\
\hline $\mathrm{N}$ & 243000 & 000 & & $\mathrm{v}$ & 00 & 9000 & 000 \\
\hline C & -3.420252000 & & & C & & & \\
\hline$c$ & -3.4 & & & & & & \\
\hline $\mathrm{F}$ & -2.787658000 & 4.96 & -2.2 & $F$ & 2.6 & -4. & 00 \\
\hline $\mathrm{F}$ & -2.793248000 & 6000 & 2.30 & $F$ & 4000 & 34000 & 3000 \\
\hline $\mathrm{O}$ & -2.451763000 & 6.235728000 & 0.00 & 0 & 1000 & -6.2 & 2000 \\
\hline C & -3.538926000 & 57000 & 0.00 & C & 000 & -7.135 & 0.000009000 \\
\hline $\mathrm{H}$ & -3.12 & 0000 & -3.9 & 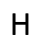 & 3.0 & -3.428173000 & -3.97 \\
\hline $\mathrm{H}$ & -3.98 & 9000 & & $\mathrm{H}$ & & 2000 & -2.6 \\
\hline $\mathrm{H}$ & -3.86 & -0.6 & $-5 . c$ & - & & & \\
\hline Ir & -3.71 & 0.3 & -0.0 & Ir & & & \\
\hline $\mathrm{H}$ & & & & y & & & \\
\hline $\mathrm{H}$ & -3 & -0.7 & & 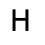 & & & \\
\hline H & -3.9 & -1.2 & & & 0 & 00 & 00 \\
\hline $\mathrm{H}$ & -4.173 & 5000 & & & 00 & -7 & 00 \\
\hline $\mathrm{H}$ & -4.17 & 3000 & -0.8 & & 00 & 19000 & 4000 \\
\hline $\mathrm{H}$ & -3.13 & 8.13 & & $\mathrm{H}$ & 00 & -8.1 & -0. \\
\hline C & 000 & -2.3 & & & 00 & 00 & 00 \\
\hline C & 3.39 & -2.00 & & C & & 0 & \\
\hline $\mathrm{N}$ & & & & 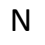 & & & \\
\hline C & & & & $c$ & & & \\
\hline C & & -0 & & $\mathrm{C}$ & & & \\
\hline C & 00 & -1. & & & 00 & 00 & 000 \\
\hline C & 3.231258000 & -2.900113000 & -1.2 & $C$ & -3.176776000 & 923123000 & 146000 \\
\hline C & 2.940339000 & -4.259263000 & -1.204299000 & & -2.863211000 & 277622000 & -1.205979000 \\
\hline C & 2.785150000 & 75000 & $0 . c$ & & -2.695833000 & 955667000 & 000 \\
\hline C & 2.943267000 & -4.258072000 & 000 & E & -2.863209000 & 4.277615000 & 206002000 \\
\hline C & 3.234282000 & 0000 & 00 & C & 76774000 & 16000 & 000 \\
\hline C & 00 & -2.2 & & $c$ & 00 & & 00 \\
\hline C & 00 & & & & & & 000 \\
\hline C & & & & & & & \\
\hline C & & & & & & & \\
\hline C & & -0.1 & & C & & & \\
\hline C & 3.804129000 & & 2.975 & & -3.803138000 & -0.163190000 & 2.978064000 \\
\hline $\mathrm{N}$ & 3.649246000 & -0.711710000 & 2027 & $\mathrm{~N}$ & -3.628169000 & 0.742317000 & 2.025427000 \\
\hline C & 3.420251000 & -1.792305000 & -6.152188 & C & -3.396271000 & 1.814593000 & -6.151199000 \\
\hline C & 3.436029000 & -1.786135000 & 61510600 & C & -3.396260000 & 814558000 & 6.151209000 \\
\hline $\mathrm{F}$ & 2.787656000 & -4.963625000 & -2.296563000 & $\mathrm{~F}$ & -2.699436000 & 978545000 & -2.298301000 \\
\hline $\mathrm{F}$ & & & & $\mathrm{F}$ & & & \\
\hline $\mathrm{O}$ & 2.451762000 & -6.235729000 & 0.002848000 & 0 & -2.340791000 & 6.245437000 & 0.000017000 \\
\hline
\end{tabular}




\begin{tabular}{|c|c|c|c|c|c|c|c|}
\hline C & 3.538925000 & -7.107769000 & 0.001658000 & C & -3.413165000 & 7.135954000 & 0.000019000 \\
\hline $\mathrm{H}$ & 3.124264000 & -3.410010000 & -3.976399000 & $\mathrm{H}$ & -3.064890000 & 3.428176000 & -3.977753000 \\
\hline $\mathrm{H}$ & 3.984440000 & 1.223575000 & -2.639943000 & $\mathrm{H}$ & -4.011468000 & -1.187691000 & -2.634849000 \\
\hline $\mathrm{H}$ & 3.865274000 & 0.696083000 & -5.059985000 & $\mathrm{H}$ & -3.889661000 & -0.663318000 & -5.057716000 \\
\hline $\mathrm{Ir}$ & 3.715366000 & -0.377076000 & -0.001667000 & Ir & -3.697178000 & 0.407768000 & 0.000001000 \\
\hline $\mathrm{H}$ & 3.134478000 & -3.406084000 & 3.977635000 & $\mathrm{H}$ & -3.064882000 & 3.428154000 & 3.977771000 \\
\hline $\mathrm{H}$ & 3.879187000 & 0.700927000 & 5.055210000 & $\mathrm{H}$ & -3.889657000 & -0.663345000 & 5.057713000 \\
\hline $\mathrm{H}$ & 3.991965000 & 1.226036000 & 2.634381000 & $\mathrm{H}$ & -4.011469000 & -1.187705000 & 3000 \\
\hline $\mathrm{H}$ & 4.173991000 & -6.97 & 00 & $\mathrm{H}$ & & & \\
\hline $\mathrm{H}$ & 4.171 & -6.976762 & -0.89944 & $\mathrm{H}$ & -4.048726000 & 7.015035000 & -0.90 \\
\hline $\mathrm{H}$ & 3.137865000 & -8.133578000 & 0.002934000 & $\mathrm{H}$ & -2.994558000 & $8.1546 \epsilon$ & \\
\hline S & -0.058221000 & 2.079554000 & 0.001493000 & $\mathrm{~s}$ & 0.025987000 & -2.096400000 & 0.00 \\
\hline C & 1.805174000 & 0.200080000 & 0.000740000 & C & -1.797440000 & -0.160062000 & 0.00 \\
\hline C & 1.603519000 & 1.576968000 & 0.000999000 & C & -1.619992000 & -1.585609000 & 0.000 \\
\hline C & 0.552067000 & -0.432819000 & 0.001384000 & C & -0.557052000 & 0.436306000 & 0.000002000 \\
\hline C & -0.552067000 & 0.432 & 86000 & C & 7051000 & -0.436306000 & 0.000003000 \\
\hline C & -1.805173000 & -0.20 & 00 & C & 000 & & 0.0 \\
\hline C & -1.603519000 & -1.5 & & C & & & \\
\hline S & 0.05 & -2.07 & 0.0 & $\mathrm{~S}$ & & & -0.0 \\
\hline$C$ & 2.725506000 & 2.4 & -0.0 & C & 000 & -2.4 & $-0 . c$ \\
\hline C & -2.725508000 & 04000 & 0000 & C & 0567000 & 000 & -0.0 \\
\hline $\mathrm{N}$ & -3.913811000 & -1.815006000 & -0.002950000 & $\mathrm{~N}$ & 000 & 000 & $-0.0 c$ \\
\hline C & -5.034927000 & -2.513573000 & -0.006384000 & C & 000 & 000 & -0.00 \\
\hline C & -5.051328000 & -3.897609000 & -0.007863000 & C & 000 & 000 & -0.000016000 \\
\hline C & -3.835667000 & -4.5 & 7000 & $c$ & 000 & & -0.00 \\
\hline C & -2.666396000 & -3.8 & -0.0 & 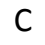 & & & 000 \\
\hline C & $2.66 t$ & & & C & & & \\
\hline C & 3.83 & 4.5 & -0.6 & C & 00 & & \\
\hline C & 5.05 & 3.8 & -0 & C & 00 & & \\
\hline C & 5.034 & 000 & -0. & C & 000 & -2.4 & -0 . \\
\hline $\mathrm{N}$ & 3.9138 & 6000 & -0.0 & $\mathrm{~N}$ & 000 & -1.7 & 000 \\
\hline C & -6.337414000 & -4.65 & 000 & C & 000 & 4.58 & 1000 \\
\hline C & 6.3374 & 4.65 & 0.0 & C & 000 & $-4.5 \varepsilon$ & -0.0 \\
\hline $\mathrm{F}$ & 7.3683 & 3.86 & -0.1 & 5 & 000 & $-3.7 \varepsilon-x-2$ & -0.0 \\
\hline $\mathrm{F}$ & 8000 & & & $\Gamma$ & & -5 & $-1 . c$ \\
\hline $\mathrm{F}$ & & & & $\mathrm{F}$ & & & \\
\hline $\mathrm{F}$ & -6 & & & $\mathrm{~F}$ & & & 00 \\
\hline $\mathrm{F}$ & -6.52 & -5.2 & 00 & $\mathrm{~F}$ & 00 & 5.3 & 000 \\
\hline $\mathrm{F}$ & -7.36 & -3.8 & -0.1 & $\mathrm{~F}$ & 000 & 000 & 000 \\
\hline $\mathrm{H}$ & -5.965252000 & -1.922805000 & 387000 & $\mathrm{H}$ & 5.983542000 & 1.864640000 & -0.000010000 \\
\hline $\mathrm{H}$ & -3.808878000 & -5.678241000 & -0.008744000 & $\mathrm{H}$ & 866400000 & 5.650553000 & -0.000020000 \\
\hline $\mathrm{H}$ & 1.693284000 & 4.36 & -0.00 & $\mathrm{H}$ & -1.738728000 & -4.369 & -0.000003000 \\
\hline $\mathrm{H}$ & 3.808865000 & 5.678240000 & -0.008725000 & $\mathrm{H}$ & -3.866399000 & -5.650553000 & -0.000018000 \\
\hline $\mathrm{H}$ & 5.965 & 00 & -0.0 & $\mathrm{H}$ & 2000 & -1.86 & -0.000013000 \\
\hline $\mathrm{H}$ & -1 & & & $\mathrm{H}$ & & & 00 \\
\hline $\mathrm{Cl}$ & & $-0.7+x-y$ & & $\mathrm{Cl}$ & & & 00 \\
\hline $\mathrm{Cl}$ & -6.1 & & & $\mathrm{Cl}$ & & & \\
\hline $\mathrm{H}$ & -4.4 & & & $\mathrm{H}$ & & & -6 \\
\hline $\mathrm{H}$ & -2.71 & 1.14 & -6.7 & $\mathrm{H}$ & 000 & -1 & 000 \\
\hline $\mathrm{H}$ & -3.115303000 & 2.841111000 & -6.294748000 & $\mathrm{H}$ & 3.086681000 & -2.861764000 & -6.295072000 \\
\hline $\mathrm{H}$ & -4.417658000 & 1.644222000 & 92000 & $\mathrm{H}$ & 4.377739000 & -1.681533000 & 6.641919000 \\
\hline $\mathrm{H}$ & -3.137413000 & 836491000 & 39000 & $\mathrm{H}$ & 3.086600000 & -2.861716000 & 6.295084000 \\
\hline $\mathrm{H}$ & -2.724104000 & 145035000 & 00242000 & $\mathrm{H}$ & 2.689086000 & -1.165929000 & 6.697681000 \\
\hline $\mathrm{H}$ & 4.402582000 & -1.657372000 & -6.64088 & $\mathrm{H}$ & -4.377739000 & 1.681552000 & -6.641914000 \\
\hline II & & & & $\mathrm{H}$ & & 1.165968000 & -6.697673000 \\
\hline 11 & 3.115279000 & -2.841090000 & 000 & $\mathrm{H}$ & -3.086614000 & 000 & 71000 \\
\hline H & 6000 & -2.8 & & $\mathrm{H}$ & 000 & 1.68 & 000 \\
\hline H & 2.724076000 & -1.1 & 000 & $\mathrm{H}$ & -3.086620000 & 000 & 86000 \\
\hline $\mathrm{H}$ & 4.417656000 & -1.644188000 & 6.639104000 & $\mathrm{H}$ & -2.689053000 & 1.165942000 & 6.697672000 \\
\hline
\end{tabular}

